\title{
BMJ Open Towards a conceptual framework of the working alliance in a blended low- intensity cognitive behavioural therapy intervention for depression in primary mental health care: a qualitative study
}

\author{
Asmae Doukani (D) , ${ }^{1}$ Caroline Free, ${ }^{1}$ Daniel Michelson, ${ }^{2}$ Ricardo Araya, ${ }^{3}$ \\ Jesús Montero-Marin (1D , ${ }^{4}$ Sarah Smith (i) , ${ }^{5}$ Arlinda Cerga-Pashoja, ${ }^{1}$ \\ Ritsuko Kakuma ${ }^{1}$
}

To cite: Doukani A, Free C, Michelson D, et al. Towards a conceptual framework of the working alliance in a blended low-intensity cognitive behavioural therapy intervention for depression in primary mental health care: a qualitative study. BMJ Open 2020;10:e036299. doi:10.1136/ bmjopen-2019-036299

- Prepublication history for this paper is available online To view these files, please visit the journal online (http://dx.doi. org/10.1136/bmjopen-2019036299).

Received 17 December 2019 Revised 18 July 2020 Accepted 07 August 2020

Check for updates

(C) Author(s) (or their employer(s)) 2020. Re-use permitted under CC BY-NC. No commercial re-use. See rights and permissions. Published by BMJ.

For numbered affiliations see end of article.

Correspondence to

Asmae Doukani;

asmae.doukani@Ishtm.ac.uk

\section{ABSTRACT}

Objectives To examine and adapt a conceptual framework of the working alliance (WA) in the context of a low-intensity blended (psychological well-being practitioner (PWP) plus computerised program) cognitive behavioural therapy intervention (b-CBT) for depression. Design Patient involvement was enlisted to collaboratively shape the design of the project from the onset, before data collection. In-depth semi-structured interviews were carried out with participants who experienced b-CBT as part of the E-compared trial. $A$ thematic analysis was conducted using a constant comparative method informed by grounded theory.

Setting Recruitment was carried out in four psychological primary care services across the UK.

Participants Nineteen trial participants with major depressive disorder who completed at least one computerised program and face-to-face session with a PWP in the b-CBT arm were recruited to the study. Results Qualitative interviews that were guided by WA theory and patient involvement, revealed four themes: (1) a healthcare provider (PWP and computerised program) with good interpersonal competencies for building a working relationship with the client ('bond'); (2) collaborative efforts between the client and the provider to appropriately identify what the client hopes to achieve through therapy ('goals'); (3) the selection of acceptable therapeutic activities that address client goals and the availability of responsive support ('task') and (4) the promotion of active engagement and autonomous problem solving ('usability heuristics'). Participants described how the PWP and computerised program uniquely and collectively contributed to different WA needs.

Conclusions This study is the first to offer a preliminary conceptual framework of WA in b-CBT for depression, and how such demands can be addressed through blended PWP-computerised program delivery. These findings can be used to promote WA in technological design and clinical practice, thereby promoting engagement to b-CBT interventions and effective deployment of practitioner and program resources.

Trial registration number ISRCTN12388725.
Strengths and limitations of this study

- Patient involvement enabled the project aims to be grounded in the needs and interests of people who have experienced mental health service-use, in order to enhance the application of the findings.

- Bordin's theory was specifically selected to examine the working alliance (WA) in blended cognitive behavioural therapy, due to the theory's comprehensive description, pan-theoretical nature, and openness to adaptation to accommodate different therapeutic formats.

- The studies' sample is limited to 19 individuals with a primary diagnosis of mild-to-moderate depression, who mostly reporting moderate-to-high WA, and were largely male, British white and university educated individuals. This thereby restricts the generalisability of our findings.

- Exposure to only one type of digital program may have influenced participants' experience of WA (eg, a computerised platform that does not work adequately might generate more data on the importance of 'ease of use', than one that does), limiting the breadth of data collected on WA.

- Efforts were made to strengthen the conceptual framework through a topic guide that was informed by Bordin's WA theory, patient involvement input and a data analysis approach which avoided surfacelevel themes specific to technological design.

\section{INTRODUCTION}

Mental health conditions impact one in six people in Europe, resulting in an estimated economic burden of over $€ 600$ billion. ${ }^{1}$ The treatment gap in the region remains high with $35 \%-50 \%$ of people experiencing mental health concerns not accessing treatment. ${ }^{1}$ The wide disparity between mental healthcare needs and access to services has prompted calls for the strategic deployment 
of technology to facilitate and expand access to mental health services at a lower cost. ${ }^{23}$ In the past decade, an increasing number of studies have investigated the efficacy of computerised cognitive behavioural therapy (c-CBT), a type of digital intervention that delivers CBT via interactive presentation features. ${ }^{4}$ The implementation of c-CBT is generally either unguided (led by a computerised program with no external support), guided (led by a computerised program and typically supported by a non-specialist facilitator) or blended (led by a therapist, incorporating a c-CBT program, or led by a c-CBT program and supported by a therapist), with the latter approach offering the highest level of human support. ${ }^{45}$

The evidence for c-CBT has demonstrated equal benefits to face-to-face CBT for a range of mental health conditions. ${ }^{4}$ However, these findings largely hold true when digital psychotherapies are guided by a human facilitator. Higher support from a therapist or another human facilitator appears to be associated with better adherence and clinical outcomes. ${ }^{6}$ The effects of human support on engagement with c-CBT raises important questions about mechanisms that support positive change. This has led scholars to consider the applicability of established mechanisms of change derived from conventional psychotherapies, to 'blended' formats. Particular interest has centred on the construct of the client-therapist alliance (the use of the 'alliance' as a singular, broadly refers to the client-therapist alliance, and not to a specific variation - eg, therapeutic alliance, working alliance, helping alliance etc - which have distinct theoretical underpinnings) ${ }^{78}$ While the concept of the alliance has taken root in a number of psychotherapeutic approaches, Edward Bordin $^{9}$ drew on their commonalities to formulate a pan-theoretical theory called the working alliance (WA) defined as:

a client seeking change and the therapist offering to act as a change agent that [incorporates] a mutual understanding and agreement about change goals and the necessary tasks to move forward these goals along with the establishment of bonds to maintain the partners' work. ${ }^{10}$ (p. 13)

Here, the 'task' refers to an agreed-upon contract that specifies the activities used to work on the client's goals. 'Goals' entails the exploration and review of what the client wants to achieve in therapy, while the 'bond' relates to the perceived compatibility between the client and the therapist, and the partnership that stems from shared activities. ${ }^{910}$ Central to Bordin's ${ }^{910}$ conceptualisation is the collaboration and consensus between the therapist and the client, in order to promote meaningful engagement in therapy.

The alliance has consistently been found to predict positive therapeutic outcomes. A keystone meta-analytic review found that the therapeutic alliance accounted for more variance $(30 \%)$ than the therapeutic technique $(15 \%)$ and therapy expectancy $(15 \%) .{ }^{11}$ This allianceoutcome relationship finding was mirrored in recent meta-analyses, one of 191 varied therapeutic studies $(\mathrm{r}=0.28$ (95\% CI 0.25 to o.30) $),{ }^{12}$ and another focusing on CBT interventions for depression $(\mathrm{r}=0.26$ (95\% CI 0.19 to 0.32$))^{13}$

A growing body of literature appears to indicate that the quality of the alliance in guided internet-based psychotherapy programmes and blended (b)-CBT may be equal to or better than traditional formats of face-to-face therapy. ${ }^{14-16}$ There is also evidence to suggest that the client-reported alliance in guided c-CBT is directly associated with treatment outcome. ${ }^{17} 18$ However, some literature appears to suggest that c-CBT may place different demands on the alliance. A narrative review evaluating WA in supported c-CBT found that while significant associations were found between the task and goals subscales of WA and treatment outcome, none was found for the bond subscale. ${ }^{18}$ Qualitative research on the alliance in unguided mental health interventions also indicate that c-CBT may offer additional alliance benefits such as higher control and autonomy. ${ }^{19} 20$

Taken together, these findings underscore the importance of developing a guiding framework for understanding the nature of WA in b-CBT, amid a gradual movement towards shared mental healthcare delivery between human practitioners and digital technology. ${ }^{21}$ Our study therefore aims to examine the WA demands through patient involvement and participant qualitative interviews, to adapt Bordin's ${ }^{9} 10$ conceptualisation of WA for a b-CBT intervention for depression. ${ }^{22}$

\section{METHODS}

\section{Patient and public involvement}

Patient advisors were enlisted at a preresearch data collection stage to collaboratively examine WA in a c-CBT program without human support. Patient advisors were not involved in the recruitment of participants or of conducting the study. Patient involvement included 11 advisors with experience of mental health service-use for mild-to-moderate depression $(n=7)$, anxiety $(n=1)$ and severe mental health conditions $(n=3)$. It should be noted that patient involvement was enlisted before the focus of the project was finalised, therefore people with a range of lived experiences were invited to be involved. Advisors attended two meetings in the summer of 2015. The first meeting consisted of a comprehensive pre-involvement preparation briefing, to provide advisors with the knowledge and skills that would enable optimal conditions for their involvment. ${ }^{23}$ Advisors were also provided with access to a c-CBT program for depression called Moodbuster (program used on the E-compared trial) ${ }^{5}$ which they were encouraged to test and review in their own time to provide context for discussion. ${ }^{23}$ Advisors voluntarily tested all components of the Moodbuster intervention between meetings. In the second meeting, advisors were split into three small focus group discussion interviews, to facilitate the sharing of personal experiences and enable a higher level of opportunities to participate. ${ }^{24}$ Discussions 
attempted to address three broad objectives, including: (1) Is WA, as defined by Bordin ${ }^{910}$ relevant in the context of digital psychological interventions? (2) What are the intrinsic WA demands of a client in relation to a digital provider? and (3) Can digital delivery offer new ways of building WA, above and beyond Bordin's ${ }^{9}{ }^{10}$ bond, goals and task? The three focus group discussions were audiorecorded using an Olympus digital voice recorder WS-852, transcribed and analysed to identify thematic patterns and themes. Patient involvement contribution was reported in line with V.2 of the Guidance for Reporting Involvement of Patients and the Public Short Form. ${ }^{25}$ Patient advisors were thanked for their contribution after their involvement and also in the acknowledgements of this paper. The results of the study will be disseminated to patient advisors via a lay summary of the research, which will be supplemented with the peer-reviewed publication.

Patient involvement was instrumental in shaping the focus of the study and in guiding participant interviews in three different ways: First, patient advisors suggested that Bordin's ${ }^{9}{ }^{10} \mathrm{WA}$, as a function of enhancing engagement, was both relevant and important in the context of a digital psychological intervention. Second, the focus of the planned qualitative research changed from exploring WA within a c-CBT intervention without human support, to exploring WA in a b-CBT format. This was in response to advisors' unanimous feedback that some WA needs (especially bond and elements of task support) could not be satisfied without human facilitation. Third, we set out to extend Bordin's ${ }^{9}{ }^{10}$ WA theory as patient advisors fed back that the c-CBT program could lead to additional alliance building and maintenance benefits.

\section{Design}

A qualitative methodology design was used to gain an in-depth understanding of participants' experience of WA in b-CBT on the E-compared trial. ${ }^{5}$ E-compared is a non-inferiority, pragmatic trial that evaluated the costeffectiveness of b-CBT for depression, when compared with usual care, across eight countries in the European region. ${ }^{5}$ Potential participants from the UK were referred from primary care services by clinical staff, if they scored 4 points or higher on the Patient Health Questionnaire-9 (PHQ-9), ${ }^{26}$ and if they were interested in receiving b-CBT for depression. The b-CBT intervention consisted of 11 blended low-intensity CBT sessions, six 30 minute sessions with a low-intensity psychological well-being practitioner (PWP), a workforce that typically provide short-term, evidenced-based treatment in line with National Institute for Health and Care Excellence (NICE) guidance in the UK, and at least five sessions at home via a synchronised computerised platform and mobile-application called Moodbuster. The treatment course spanned across 11 weeks. There were four mandatory core modules of CBT on the computerised platform (psychological education, behavioural activation, cognitive restructuring and relapse prevention) and two optional modules (physical exercise and problem solving) that were completed autonomously at home. The low-intensity PWP in the clinic encouraged participants to use the computerised program in different ways. The PWP could introduce modules, review the client's progress on the modules, or guide the client on the use of specific modules. Face-to-face sessions in the clinic were alternated with Moodbuster sessions away from the clinic, however there was flexibility in the sequence of the delivery mode and the order in which the modules were completed, including opportunities for the PWP to use bespoke tasks. Additional information about the trial and the b-CBT intervention can be accessed from the E-compared trial protocol by Kleiboer $e t a l^{5}$

\section{Participants}

E-compared participants from the UK were invited to take part in qualitative interviews. Trial participants aged 18 years or older with a clinical diagnosis of major depressive disorder were enrolled in the study. ${ }^{5}$ People with substance abuse, suicidal tendencies, other severe psychiatric disorders, cognitive disability or people who had insufficient knowledge of English were excluded. Psychiatric diagnoses were confirmed by the MINI International Neuropsychiatric Interview V.5.0. ${ }^{27}$ E-compared trial $^{5}$ participants who: (a) provided written consent to the qualitative interviews when they enrolled on the trial $(\mathrm{n}=101)$; (b) were randomised to the b-CBT arm $(\mathrm{n}=49)$ and (c) had completed at least one computerised module and face-to-face session $(n=42)$ were purposively sampled to be representative of the b-CBT arm, in relation to their sex, age and recruitment site. ${ }^{28}$ Altogether, 26 out of 42 people were invited to take part in the qualitative study, with 19 reconsenting to participate. Reasons for non-consent included scheduling conflicts $(n=2)$ nonresponse to invitation $(n=4)$, and change in eligibility status due to erroneous information about treatment arm allocation $(\mathrm{n}=1)$. This case was discovered during the interview, and corroborated with the E-compared UK trial manager after the interview. Data for this participant were not analysed.

\section{Procedure}

E-compared participants were invited to take part in faceto-face individual semi-structured qualitative interviews, at least 2 weeks after they completed their course of therapy on the trial. This was to provide participants with enough time to reflect on their experience of the b-CBT intervention. Potential participants were invited to take part in interviews about their experience of b-CBT, and were emailed a patient information sheet following their initial correspondence with the research team. Participants were provided with at least 48 hours to read and consolidate the information, before they were followed up and booked in for a qualitative interview at an acceptable time and place. Written consent for their participation, and permission to audio record interviews, were sought again prior to starting the interviews. Participants were also reminded of their right to withdraw at any time and without giving a reason. Data collection took place 
until saturation was reached. ${ }^{28}$ The study adopted Corbin and Strauss's definition of saturation, which is described as the point where further data collection becomes 'counterproductive', and where 'new' themes do not add anything to the overall narrative of the story. ${ }^{28}$ Saturation was monitored through writing memos after each interview, in which information on both exisiting and novel emerging themes from the interview were noted. ${ }^{28}$

\section{Measures}

Data collected on self-reported WA and depression questionnaires on the E-compared trial ${ }^{5}$ were reported to further describe participant characteristics (in addition to sociodemographic data) to provide insights into the level WA and depression symptoms experienced by participants on this study. Self-reported WA was assessed through the Working Alliance Inventory Short FormClient (WAI-SF-C) ${ }^{29}$ Scores for the 12 items on WAI-SF-C range between 12 and 60 . Scores were divided into three groups to produce a low-range (12-28), medium-range (29-44) and high-range (45-60) to indicate the level of WA reported by each participant. Higher scores indicate better WA. Self-reported depression was assessed through the PHQ-9. ${ }^{26}$ Scores for the nine items on the PHQ-9 range between 0 and 27. Higher scores indicate more severe symptoms. Data were collected during the trial's 3 months follow-up assessments. ${ }^{5}$

\section{Guiding framework}

Our study adopted Edward Bordin's ${ }^{910}$ theory to examine WA in the context of b-CBT for three reasons. The first relates to the generalisable nature of the theory. While the concept of the alliance stemmed from psychodynamic theory in 1912, it has since been incorporated in various therapeutic approaches, leading to heterogeneity in the way the concept is defined. ${ }^{12}$ In 1979 , Bordin $^{910}$ attempted to unify the way the alliance is defined, by proposing a pan-theoretical conceptualisation $^{9}$ that drew on the key features of all therapeutic approaches. ${ }^{12}$ Second, Bordin's ${ }^{9}{ }^{10}$ theory is operationalised as task focused, ${ }^{12}$ and therefore offers a suitable fit for task-orientated psychological approaches such as $\mathrm{CBT}^{30}$ Third, the theory is open to adaptation. Bordin ${ }^{910}$ suggested that while a pan-theoretical approach allowed the basic measurement of the bond, goals and task to produce beneficial therapeutic change, he also suggested that the ideal alliance 'profile' is likely to be different across therapeutic approaches and interventions. ${ }^{9} 1012$

\section{Data collection}

Data collection took place between October 2016 and July 2017 across four primary care mental health services in the UK. Qualitative interviews were adopted to enable a detailed examination of the participant's personal experiences and perspectives of WA within the context of receiving b-CBT. The study predominately included a deductive approach to exploring WA that was guided by Bordin's ${ }^{910}$ theoretical framework, while remaining open to novel or unexpected new themes. On average, participant interviews lasted around $47 \mathrm{~min}$. Interviews were conducted in a confidential setting within a university campus or the health service which the participant was recruited from. All interviews were audio-recorded using an Olympus digital voice recorder WS-852 and transcribed to produce orthographic verbal verbatim. AD (female) conducted the qualitative interviews, was a $\mathrm{PhD}$ candidate with experience of conducting and analysing qualitative data. Semi-structured interviews with a conversational technique were used to achieve a balance between the need for consistency of questioning across participants, and the ability to explore topics that are important to the participant. During interviews there was also scope to allow topics covered to evolve iteratively based on the emerging data. ${ }^{28}$ The development of an interview topic guide was guided by patient involvement input and WA theory. ${ }^{9}{ }^{10}$ The initial topic guide was used to suggest topics of discussion, and not as a definitive framework to limit conversations. As the data collection progressed, the topic guides evolved iteratively based on emerging themes. Subsequent interviews were therefore influenced by interviews that previously took place, providing opportunities to validate and refute interpretations. ${ }^{28}$

\section{Data analysis}

A preliminary data analysis took place alongside early interviews, allowing subsequent interviews to progress iteratively. ${ }^{28}$ Memos were written after each interview, to aid the preliminary analysis and iterative adaptation of the topic guide and to propose possible relationships between codes. Thematic analysis was adopted due to the theoretical flexibility, as well as the 'thick descriptions' afforded by the approach. ${ }^{32}$ The data analysis incorporated a constant comparative method from grounded theory, to enable the analyst to search for new theoretical models that are grounded in empirical data and to enhance the trustworthiness of data. ${ }^{28}$

The lead analyst (AD) commenced the data analysis by reading through the transcripts, while listening to the audio-recording and reading the corresponding memos. The analyst then actively re-read the data, searching for meaning and noted down initial concepts. Data were coded line-by-line. Codes were generated by searching for interesting features across the entire dataset and collating data relevant to each code segments. The emerging codes were clustered into categories and labelled thematically. Once the data were initially coded and collated, the analyst commenced searching for themes that were compatible with Bordin's ${ }^{9} 10$ WA theory and patient involvement input, while also searching for novel alliance concepts. Themes were located at a latent level, to delve beyond the semantic content of the data, to identify and examine underlying ideas, assumptions, conceptualisation and ideologies that theorise semantic content of the data. ${ }^{32}$ The initial codes were gradually merged into broader categories through comparison across transcripts, to identify overarching themes. The themes were 
then reviewed to ensure that the codes cohere together meaningfully, while maintaining a clear and identifiable distinction with no overlap between the themes. Finally, the themes were reviewed to consider their relationship to the overall thematic map. Once a thematic 'map' was identified, the findings were developed into a conceptual framework of WA in b-CBT. ${ }^{32}$

Two other members of the research team (CF and DM), who are highly familiar with qualitative methodologies and Bordin's ${ }^{9}{ }^{10}$ WA theory, read through $20 \%$ of all transcripts and reviewed all supporting quotes across all phases of the analysis, so that close to half of the transcripts were reviewed. Discrepancies were discussed and reconciled. The final framework was discussed and revised over eight meetings. The entire coding process was performed using the NVivo V.11 data analysis software package. Supporting quotes were anonymised to ensure that participants and their PWP could not be identified.

To ensure the final conceptual framework accurately reflected WA (a 'therapeutic process') was not confounded with early manifestations of 'treatment outcomes' we defined 'therapeutic processes' relevant to WA, and the 'treatment outcomes' associated with $\mathrm{CBT}^{29}$ 'Therapeutic process' was defined as 'actions, experiences and relatedness of the client and the therapist in therapy sessions...' ${ }^{33} \mathrm{We}$ a priori extended the use of the term 'therapy session' to include face-to-face and digital delivery in the context of blended therapy. Horvath $e t a l^{29}$ noted three ways of defining the outcome in psychotherapy including: (a) the core value attributed to the outcome by the client, (b) the importance of the outcome in the theoretical framework of the therapist, and (c) the utility of the outcome (eg, the technique) to promote other outcomes that are valued. We defined outcome in relation to definitions $b$ and $\mathrm{c}$ to enable a standardised definition that does not vary from client-to-client (ie, definition a). We a priori defined the outcomes of CBT as the alleviation of distress (b) through helping the client to develop more adaptive cognitions and behaviours (c). ${ }^{30}$ The final conceptual framework was reviewed in light of the aforementioned definitions by members of the research team. Themes and subthemes that were judged to correspond with the definition of 'treatment outcome' were removed. We used the Standards for Reporting Qualitative Research checklist when reporting our findings. ${ }^{34}$

\section{RESULTS}

\section{Description of sample}

An exploration of WA in b-CBT was undertaken through 19 qualitative interviews with participants who experienced b-CBT in the treatment arm of the E-compared trial. ${ }^{5}$ Participants were aged between 19 and 67 years (mean=34.47 years, $\mathrm{SD}=14.44$ years), were largely male $(n=13)$, white British or white other $(n=12)$ and university educated $(n=12)$ (full sample characteristics are presented in table 1). All interviews were conducted faceto-face apart from one, which was completed by phone.
Table 1 Sample characteristics of participants who took part in the qualitative interviews $(n=19)$

\begin{tabular}{|c|c|}
\hline Characteristics & $\begin{array}{l}\text { Mean (SD) or } \\
\text { percentage }(n)\end{array}$ \\
\hline Age in years & $\begin{array}{l}34.47(14.44), \text { range } \\
19-67 \text { years }\end{array}$ \\
\hline Gender (male) & $69 \%(13)$ \\
\hline \multicolumn{2}{|l|}{ Marital status } \\
\hline Divorced & $5 \%(1)$ \\
\hline Living together & $11 \%(2)$ \\
\hline Single & $63 \%(12)$ \\
\hline Married & $21 \%(4)$ \\
\hline \multicolumn{2}{|l|}{ Highest educational level completed } \\
\hline Secondary school, equivalent & $11 \%(2)$ \\
\hline Colleague, equivalent & $26 \%(5)$ \\
\hline University degree or higher & $63 \%(12)$ \\
\hline \multicolumn{2}{|l|}{ Ethnicity } \\
\hline British white or white other & $63.1 \%(12)$ \\
\hline $\begin{array}{l}\text { Black/African/Caribbean/Black } \\
\text { British }\end{array}$ & $5.3 \%(1)$ \\
\hline $\begin{array}{l}\text { Asian or Asian British (any other } \\
\text { Asian) }\end{array}$ & $21 \%(4)$ \\
\hline Mixed or multiple ethnic group & $5.3 \%(1)$ \\
\hline Other & $5.3 \%(1)$ \\
\hline \multicolumn{2}{|l|}{ Intervention completion level ${ }^{\star}$} \\
\hline Completed course of b-CBT & $63.2 \%(12)$ \\
\hline Incomplete course of b-CBT & $36.8 \%(7)$ \\
\hline WAI-SF-C $†$ & $\begin{array}{l}46.29(\mathrm{SD}=10.21), \text { score } \\
\text { range } 27-60(17)\end{array}$ \\
\hline High WAI-SF-C & Score range 47-60 (10) \\
\hline Medium WAI-SF-C & Score range $31-41$ (6) \\
\hline Low WAI-SF-C & Score 27 (1) \\
\hline No score & $(4)$ \\
\hline PHQ-9 & $\begin{array}{l}7.8(6.87), \text { score range } \\
1-22(n=18)\end{array}$ \\
\hline
\end{tabular}

*Intervention completion level: a complete course of b-CBT refers to the completion of four mandatory Moodbuster modules (psychological education, behavioural activation, cognitive restructuring and relapse prevention), while an incomplete course of b-CBT course refers to the non-completion of the four mandatory Moodbuster modules.

†Four participants did not provide data for this questionnaire during their 3-month follow-up assessment.

b-CBT, blended plus cognitive behavioural therapy; PHQ-9, Patient Health Questionnaire-9; WAI-SF-C, Working Alliance Inventory Short Form-Client.

Saturation appeared to be reached by the 16th interview. Another three interviews were carried out to ensure that the selected saturation cut-off point had been accurately identified and to further validate interpretations. tables $2-4$ show that the main themes were endorsed by $89 \%-100 \%$ of participants, indicating that the selected saturation cut-off point was sufficient. 
Table 2 Theme 1, bond subtheme descriptions and supporting quotes

\section{Theme, percentage of sample endorsed $(n)$ and} description

Theme 1: bond, 89\%, (17)

\subsection{Feeling understood, $74 \%$ (14)}

The PWP's ability to make the client feel understood.

This requires the PWP to closely listen to the client, comprehend what is being said and demonstrate empathic awareness and insight into the client's concerns.

\subsection{Genuineness, 32\% (6)}

The PWP's efforts to help the client, that are perceived as genuine and authentic, as opposed to procedural or routine.

\subsection{Partnership, $74 \%$ (14)}

The ability of the client and PWP to achieve a working relationship that is akin to a friendship. Such a partnership is characterised by trust, empathy, feeling liked and feeling cared for.

\section{Supporting quotes}

P12, high-range WAI-SF-C score: "My therapist did make a real effort to try and get to know me, try to maybe get to know what made me tick and why I was feeling how I did, rather than just assuming this is what you need without ... taking into account maybe what I as a person, personally needed".

P9, Iow WAI-SF-C score: "To be honest, I kind of felt like she (PWP) was very fake...Every time l'd say something there would be an, ahh, it just felt not genuine at all, that she was just saying it because she thought I felt down..."

P12, high-range WAI-SF-C score: "I feel like she, as I said earlier, took the time to get to know me and ... what I was currently doing, so it did feel like she kind of knew me on an individual level, rather than just being the patient".

PWP, psychological well-being practitioner; WAI-SF-C, Working Alliance Inventory Short Form-Client.

\section{Conceptual framework of WA in b-CBT}

A thematic analysis with a constant comparative method $^{32}$ revealed multifaceted WA demands which show that the work of building WA in b-CBT involved a symbiotic effort by the PWP and the digital program, to actively engage the client to meaningful therapeutic activities and to promote self-discovery and commitment to the intervention. Such demands can be grouped into four overarching WA themes, (1) 'bond', (2) 'goals', (3) 'task' (in line with Bordin's ${ }^{9}{ }^{10}$ WA theory categories $)^{9} 10$ and (4) 'usability heuristics' (a newly emerging theme) (see figure 1 for a summary of the main themes and subthemes).

\section{Theme 1: bond}

The 'bond' is defined as a set of mental healthcare provider (including both the PWP and computerised program) competencies that enable a working relationship to be established and maintained with a client. Participants unanimously reported that a human therapist was the most important facilitator for building the bond in a b-CBT context. This was because participants valued qualities of 'humanity' and 'responsiveness' attributed to a human therapist. Through a process in which participants appeared to compare and contrast the strengths of the digital program with the PWP, most participants questioned the 'meaningfulness' of interacting with a digital platform that was incapable of understanding or responding to a client's needs as demonstrated by the following quote:

an app is like a machine, it's not personal at all. I think it's good to have some element[s] of talking to a human about this kind of thing because I think you want reassurance as well, which you wouldn't get from an app and if you did it would just be responses built in. (P8, low-range WAI-SF-C)

Data from participant interviews revealed three broad PWP attributes considered to be important for the bond building process, namely the mental health providers' ability to effectively demonstrate their understanding of their client's struggles and needs (subtheme 1.1); convey that they are genuine in their endeavours towards the client (subtheme 1.2) and forge a working partnership founded on friendliness, feeling cared for, empathy and trust (subtheme 1.3) (see table 2 for subtheme descriptions and supporting quotes). Some participants elaborated on these concepts further to unearth granular insights of what it means to be in the presence of a PWP. Visually observing a PWP's non-verbal cues was reported to be especially important for gauging abstract relational concepts such as empathic understanding (subtheme 1.1), and genuineness (subtheme 1.2). The recognition of positive non-verbal cues appeared to increase congruence between the PWP and the client (subtheme 1.3) throughout the course of therapy:

[During telephone therapy] he was like ' $\mathrm{mm} \mathrm{hm}$, go on...so how do you feel?' I can't see his face. I don't know what he was thinking. I can't feel him. But during face-to-face [sessions] I think when I talk about something I can notice, his or her like facial expression. I know he's listening ...That make[s] me feel like talk[ing] more. (P14, WAI-SF-C score not available [WAI-SF-C scores are unavailable for participants who did not complete their online 3-month follow-up assessments on the E-compared trial])

\section{Theme 2: goals}

'Goals' refers to the collaborative work between the PWP, the client and the digital program, to appropriately identify what the client hopes to achieve through therapy (68\% of sample endorsed the 'goals' theme, $\mathrm{n}=13$ ). While 'goals' emerged as a distinct factor, it also appears to be interrelated with the 'task', thereby playing a fundamental 
Table 3 Theme 3, task subtheme descriptions and supporting quotes

\section{Theme, percentage of sample endorsed $(n)$ and} description

\section{Supporting quotes}

Theme 3: task 100\%, (19)

Activity-based task, 100\% (19)

\subsection{Personalisation, 95\% (18)}

The level at which a client is able to tailor the therapeutic task to their individual needs. A nonpersonalised digital intervention was reported to negatively impact engagement. The PWP in blendedtherapy can play an important role in making a generic intervention (ie, computerised CBT) as more personalised.

\subsection{Usefulness, 95\% (18)}

A useful task was defined as one that promotes new learning, reflection and is effective in creating desired change in the client's life.

\subsection{Complementary, $84 \%$ (16)}

The ability to experience complementary tasks in face-to-face therapy and on the digital platform as continuous and cohesive, as opposed to stilted and disjoint. Knowing what to expect from the respective components of blended therapy was reported to help the client optimise the benefits sought from different components of therapy.

Responsive support task, 100\% (19)

\subsection{Accountability, $79 \%$ (15)}

The availability of a figure of authority that the client can (positively) feel responsible towards, as a means of garnering motivation to work on therapeutic activities. For the process of accountability to positively impact the client's motivation, a PWP is required to demonstrate their knowledge of the client's progress and provide feedback accordingly.

\subsection{Guidance, $89 \%$ (17)}

The provision of guidance and reassurance on the therapeutic tasks by a PWP. The PWP's intuition, expertise, interpretation and foresight is especially considered as helpful in addressing salient issues that would not have otherwise been communicated by the client.

\subsection{Expression of feelings $100 \%$ (19)}

The client's expressed need to speak to another human being, in order to communicate issues that are pertinent to their treatment journey. In order for the client to optimally benefit, clients require the PWP to dedicate a sufficient amount of time for the activity. The amount of time required by each person appears to vary in relation to pretherapy expectations and symptom severity.
P12, high-range WAI-SF-C Score: "I think it's a bit more personalised, because I would say while the E-Compared is good, it is still, it is to an extent generic, because it can't kind of know every single person that's watching the video, so whereas the therapists can kind of get an idea of you, your story, your journey, what's maybe led you to kind of this maybe relapse, or for you to be feeling the way you are, and you can't maybe get that from a computer... Whereas if I'm hearing it from the person, I'm going to take a bit more notice, but then if I'm just hearing it from the computer, where it will say that to everyone watching the video".

P4, medium-range WAI-S-C score: "But like the modules themselves, feelings-wise they were often quite helpful for clarifying stuff. Like I usually came out the other end feeling better or more kind of composed... it would kind of shape how I was seeing things. So like if I, you know learnt about thought distortions, l'd kind of go in with that knowledge and be able to kind of talk about it..."

P16, medium working alliance: "I was finding it really hard to leave the house so that whole thought of going to therapy was quite difficult in the very beginning, so it did take me a couple of sessions to really start talking to (therapist) and opening up but because I had this online support I found it easier to open up to (therapist) so maybe instead of you know, two sessions it would have taken four or five". 
Table 4 Theme 4, usability heuristics, subtheme descriptions and supporting quotes

\section{Theme, percentage of sample endorsed $(n)$} and description

\section{Supporting quotes}

Theme 4: usability heuristics, $100 \%$ (19)

4.1. Accessibility, 95\% (18)

The ability of a client to access the digital intervention at a time and place of convenience. Higher accessibility provides opportunities for the client to review and reflect on what has been learnt at a deeper level.

4.2. Interactivity, $63 \%$ (12)
An interactive digital program that is able
to react to the clients input, to produce
feedback. Interactive activities were
perceived as more enjoyable, and promoted a
degree of accountability.

\subsection{Ease of use, $63 \%$ (12)}

The ease of use of the digital interface is described as a well-functioning, intuitive, digital interface which enables optimal access to the therapeutic task.

\subsection{Aesthetic appeal, $21 \%$ (4)}

The appearance or appeal of the digital interface is a factor that clients use to judge the credibility of the digital intervention and which could impact their engagement to the therapeutic task.

\subsection{Self-directed, $79 \%$ (15)}

The process of taking responsibility for one's own behaviour and well-being, appears to instil clients with a sense of independence and control.
P10, high-range WAI-SF-C score: "Being on your own you know, in your own time and in your own safe place, your blanket, whatever you call it just allowed me personally just to open up and look at it, and then going from the start of the process to the end, ... thinking positively, looking at your behaviours, looking at adding little things in and then the exercise at the end, rewarding yourself for just achieving things what I felt at the time were trivial made everything different".

P6, high working alliance: "One thing immediately comes to mind, it has to be a bit more interactive I think. The client shall we say, as well I feel should be given more feedback, the results, you know when you're scoring yourself on those, what that's about you know, how do they interpret that score, when you're putting your mood in on the smartphone, what's that about you know, who's looking at that, who's interpreting that".

$P 2$, high-range WAI-SF-C score: "It was really nice, I thought it was really, well very well presented I would say, and everything was just there, like for easy viewing, so you didn't have to like go through like folders or like go deeper into the website, like it was just there, and you know, I could just easily click on what I needed to do and just follow the instructions set out on the exercises".

P13, medium-range WAI-SF-C score: "Yeah, and actually it became quite a bit of work just keeping up with the calendar, sort of, I found it a bit clunky, but then I worked in I.T for sixteen years..."

P3, medium-range WAI-SF-C score: "Other times it was good kind of to do a time and also independence, kind of learning to do stuff without a therapist there...I quite liked that I could, I don't know for me because it, I suppose it ties back into the independence thing, but because I was doing it on my own I almost proved I could do it on my own... because I feel like sometimes with a therapist you almost become like dependent on them or, it's like being taught something, when you're like dependent on the teacher".

WAI-SF-C, Working Alliance Inventory Short Form-Client.

role in framing activity-based tasks and maintaining the client's motivation to work towards creating change.

The goal setting actually was something that I spoke to [the PWP] quite a bit about in the session [...] I was then like 'God well what are my goals? [...] what, where am I exactly going? (P5, higher-range WAISF-C score)

\section{Theme 3: task}

The 'task' refers to the careful selection and acceptability of the therapeutic activities prescribed to address the client's presenting symptoms ('activity-based task'), and the degree to which the support received by the healthcare provider on these activities is responsive ('responsive support').

The defining features of 'activity-based task' refers to the client's ability to work on tasks that are: personalised and acceptable for addressing the client's therapy goals (subtheme 3.1); useful in promoting new learning, insights and reflection (subtheme 3.2) and are complimentary across both modes of delivery (subtheme 3.3). The defining features of 'responsive support' relate to the provider's (largely referring to the PWP's role) ability to appropriately respond to a range of clients' expressed and unexpressed needs to maintain accountability (subtheme 3.4); provide activity-based guidance (subtheme 3.5 ) and a safespace for clients to express their feelings and emotions (subtheme 3.6) (see table 3 for subtheme descriptions, and supporting quotes).

The majority of participants noted the importance of experiencing the therapeutic activity as complementary across modes of delivery (subtheme 3.3). Some participants elaborated that an initial step to achieving an effective symbiotic delivery was to provide the client with an understanding of how the PWP and digital delivery contributed towards their treatment both distinctively and collectively.

Our findings also suggested that c-CBT appeared to positively impact the client-PWP WA, through increased 


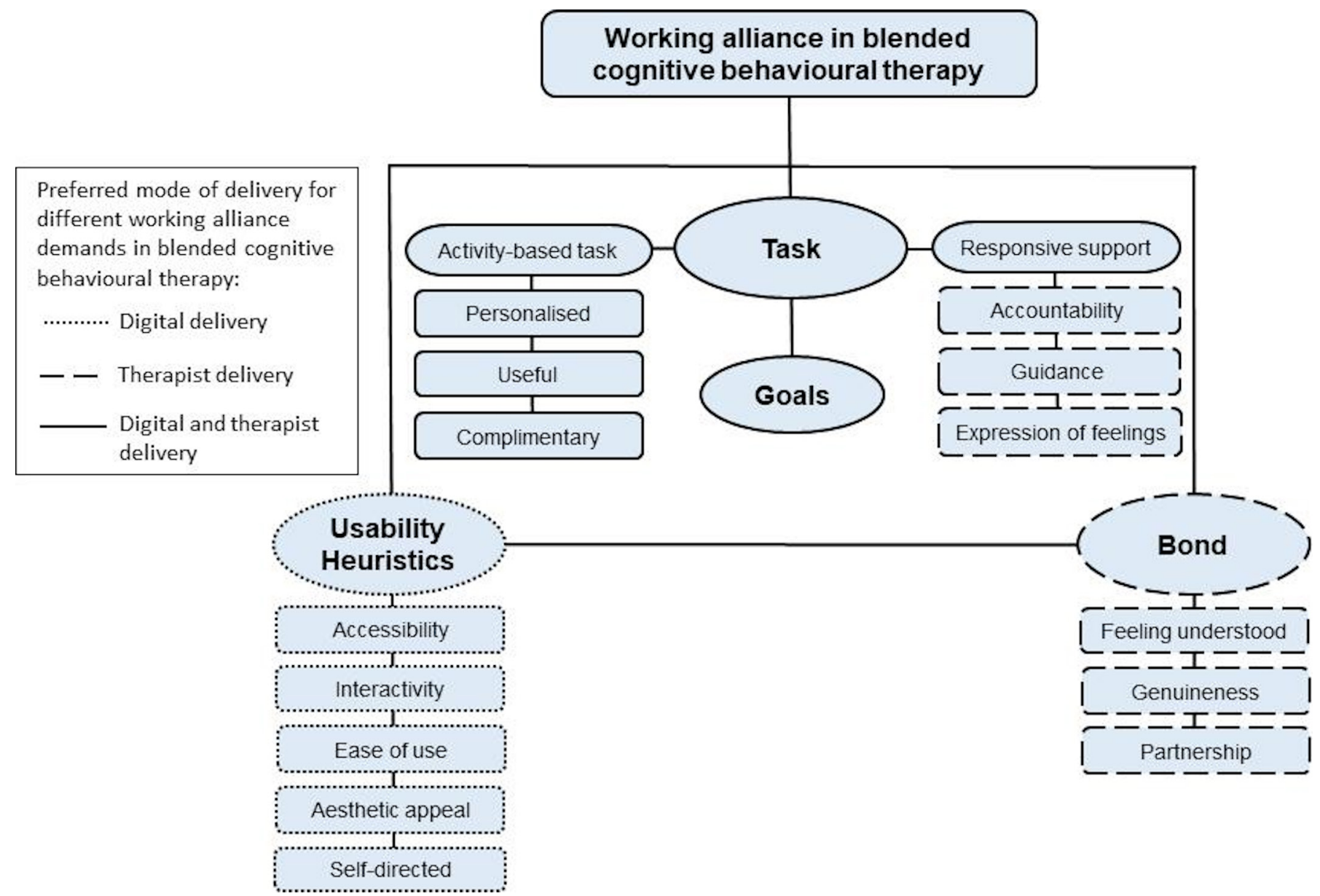

Figure 1 Participant reported working alliance demands in a blended cognitive behavioural therapy intervention.

opportunities to reinforce what was learnt through the digital platform, with a PWP, and vice versa, for instance:

Well I think it gave you something to do over and above the face-to-face... having the modules to go through, it reinforces what you're talking about faceto-face and makes it easier to understand. It's, that repetition thing isn't it where you learn by repetition basically and that's how I saw it working. (P17, WAISF-C score not available)

\section{Theme 4: usability heuristics}

The final alliance building theme identified is, "usability heuristics', which refers to the process of predominantly using digital technologies to promote active engagement, self-discovery and autonomous problem solving in b-CBT. This category is a novel component to Bordin's ${ }^{9} 10$ theory. Features that enable 'usability heuristics' include ubiquitous digital technologies that: increase access and immediacy to the therapeutic task (subtheme 4.1), appropriately respond to the client's input (subtheme 4.2), are easy to use (subtheme 4.3) have aesthetic appeal (subtheme 4.4) and promotes self-directed therapy (subtheme 4.5) (see table 4 for subtheme descriptions and supporting quotes).
While PWP competencies emerged as the most important facilitator for building the alliance, almost all participants expressed that they preferred blended CBT to face-to-face alone. Some participants elaborated that their ability to access the intervention at a time and place of convenience (subthemes 4.1) further bolstered their engagement to therapeutic activities (theme 2). Participants who reported a high technological affinity suggested that the appearance (subtheme 4.4) and ease of use (subtheme 4.3) of the interface impacted their perceptions of the digital program's credibility and therefore, their desire to engage in treatment activities.

Almost all participants reported that the digital program provided them with the tools to initiate treatment independently (subtheme 4.5), with some participants noting that they continued to use the digital program as a means of maintaining therapeutic gains once their therapy course had ended. Here, autonomous completion of the therapeutic task was described as a secure-base that allowed clients to progressively explore self-directed therapy:

it kind of reminds me of Winnicott and the Secure Base in Attachment theory in psychology, that you know, children become securely attached if they have a secure base in terms of the home and the parents 
that they can come back to, so they can go off and explore the world confidently in the knowledge that they can come back to security, and that, that helps them to develop-and it's kind of like that, I feel, with having that Moodbuster resource (digital program) there, that you can keep coming back to it ... there is a lot in there and you can keep going back and it's a sort of source of strength really. (P10, higher-range WAI-SF-C score)

Participants suggested that the blended approach prepared the client to engage in autonomous self-directed therapy, through a process of supervised autonomy.

\section{DISCUSSION}

\section{Statement of principal findings}

The results of the study present a preliminary conceptual framework of WA in b-CBT. It can be seen that Bordin's ${ }^{910}$ 'bond', 'goals' and 'task' appear to be relevant in b-CBT, however the priorities of WA demands have shifted to meet the client needs within a blended format. Moreover, an entirely new category 'usability heuristics', emerged as a novel means of promoting WA through a process of self-directed discovery and autonomous problem solving. Participants also explained that different WA demands were met by the PWP (eg, client-provider bond, responsive support) and the digital program (eg, upholding goals, task and promoting usability heuristics).

\section{Strengths and limitations of the study}

To our knowledge, this study is the first to provide an account of WA in b-CBT, and insights into how different delivery roles within a blended format are used to meet different WA demands. This is especially important given that digital technologies are increasingly being used to treat mental health conditions, ${ }^{4}$ and that WA plays an important role in promoting positive therapeutic change. ${ }^{12}$ The design of our study had two key strengths. First, we used the most comprehensive and commonly used theory of the alliance ${ }^{35}$ Second, involving patient involvement enabled the project to be grounded on the needs and interests of people who have experienced mental health conditions and service-use, thereby enhancing the application of our findings. ${ }^{23}$ There are also several limitations to be noted. Our study does not include the PWP's perspective, which may have provided additional insights on WA in b-CBT, ${ }^{18}$ however, this will be explored in a separate paper. Our sample was limited to 19 individuals with a primary diagnosis of mild-to-moderate depression and who mostly reported moderate-to-high WA, were largely male, British white or white other and university educated. This thereby limits the representativeness of people seeking treatment in the $\mathrm{UK}^{36}$ and restricts the generalisability of our findings. Exposure to only one type of digital program, may have influenced participant's experience of WA. For instance, a computerised platform that does not work adequately might generate more data on the importance of 'ease of use' than one that does. Some of these issues were pre-empted ahead of the study. Efforts were made to strengthen the conceptual framework in two ways. First, emerging participant data were guided by key literature on the alliance and patient involvement input. Second, our qualitative data analysis avoided the use of surface-level themes, such as specific technological design. Instead, latent thematic analysis was used to unearth underlying psychological processes. $^{32}$

\section{Strengths and weaknesses in relation to other studies, discussing important differences in results}

Participants reported that, while it was essential for therapeutic activities to be complimentary between modes of delivery, they also suggested that modes of delivery can uniquely meet different WA needs. For instance, participants unanimously fed back that the PWP played an essential role in establishing the 'bond'. The importance of the practitioner's role in supporting digital interventions is well documented in the literature. ${ }^{6}$ A 2018 study evaluating the relationship between the client, the human provider and their c-CBT program found that participants rated their overall treatment approach higher when they experienced c-CBT that was guided by a human provider compared with c-CBT that was unguided. $^{7}$ Another study evaluating the expectations of clients and practitioners in a c-CBT intervention for depression found that personalised interactions with a therapist were key for engagement. ${ }^{37}$ When attempts were made to unpack the importance of the therapist's role, participants suggested that the PWP's physical presence facilitated the PWP's propensity to convey important features of the bond (subthemes 1.1-1.3) through verbal and non-verbal communication. This aligns with early psychotherapy research by Karl Rogers, ${ }^{38}$ who proposed that a therapist's ability to display active listening (empathic understanding, unconditional positive regard and congruent behaviour) was important for positively changing the impressions of the client's perceived negative experiences. Neuroscientific research evaluating the impact of active listening, suggested that the participant's recognition of active listening behaviour in another, can positively change the appraisal of an emotional episode and increased positive impressions of the active-listener. ${ }^{39}$ These findings appear to be unique to human-to-human interactions. One study assessing the therapeutic alliance in a digital mental health mobile application for psychosis found that the anthropomorphizing of digital devices was not accepted by clients or mental health practitioners. ${ }^{20}$ Given that little gains have been made to effectively deploy emotional artificial intelligence, a tool that is required for the effective biomimicry of human beings in the digital space, ${ }^{40}$ the exclusion or non-effective deployment of a human provider in digital psychological interventions may therefore compromise the quality of WA.

On the other hand, participants reported that while the PWP was essential for the effective delivery of CBT, they 
preferred blended delivery compared with PWP delivery alone. Almost all participants reported WA benefits, in the form of engagement through desired opportunities to engage in self-directed therapy (ie, 'usability heuristics'). Our findings are echoed in the digital mental health userexperience and the alliance literature, which indicate that digital psychotherapy can enhance the client's perceived control, autonomy and feelings of empowerment, when sufficient human support is provided. ${ }^{20}{ }^{41}$ The findings of our study suggest that the impact of digital delivery cannot be removed from the formation of the WA. For example, a digital program that was perceived as noninteractive appeared to limit engagement with 'activitybased task'. Given that the program plays an important role in delivering and engaging the client to 'activity-based task', we argue that the inclusion of program factors that uphold existing alliance structures should therefore be accounted for in the WA framework. Our findings align with Bordin's ${ }^{9} 10$ conceptualisation of WA, in which he proposed that the therapeutic tool cannot be disentangled from the means in which the alliance is built. This therefore suggests that the client-program WA may have an impact on the client-PWP WA, and vice-versa, contrary to research suggesting that the contribution of a digital program to WA is independent and additive. ${ }^{7}$

The 'task' appears to play a central role in b-CBT, as initially theorised by Bordin. ${ }^{9}{ }^{10}$ Our findings address Bordin's ${ }^{10}$ call to distinguish between the task that is in service of 'building WA' (responsive support) and the task in the service of 'change' (activity-based task). While many of the 'task' subthemes appear to be novel to Bordin's ${ }^{9} 10$ theory, with the exception of complementary tasks (subtheme 3.3), all other 'task' subthemes are in fact implicit in his broad conceptualisation. The integration of technology in psychotherapy has prompted a re-evaluation of the demands placed on WA by a blended psychotherapeutic format. For example, the concept of accountability is implicit and forms one of many appendages associated with the PWP's role in building and maintaining WA. However, this concept has been propelled to the forefront as an essential ingredient for maintaining the alliance in b-CBT, in line with the 'supportive accountability' model for e-health proposed by Mohr et al. ${ }^{42}$

While 'bond', 'goal', 'task' and 'usability heuristic' emerged as distinct themes, the 'goals' theme appears to be interlinked with 'task'. The data from the qualitative interviews indicated that the 'goals' theme was grounded in goal-setting activities. This however diverges from Bordin's ${ }^{10}$ description of the 'goals', which appears to move further, to address the PWP's efforts to unearth the core struggles that have bought the client to psychotherapy in great detail. ${ }^{10}$ One possible reason for our findings may be explained by the time-lag between the assessment and the first therapy session, which may have led participants to only focus on their course of b-CBT and not the proceeding assessment where more in-depth explorations of the client's struggles and goals may have taken place. On the other hand, our study is not the first
Box 1 Top 10 research priorities for digital technology in mental healthcare, identified by the priority setting partnerships ${ }^{7}$

Q1. What are the benefits and risks of delivering mental healthcare through technology instead of face-to-face and what impact does the removal of face-to-face human interaction have?

Q3. How can treatment outcomes be maximised by combining existing treatment options (medication, psychological therapies, etc) with digital mental health interventions.

Q8. Can the common elements of therapy (eg, empathy, gestures, nonverbal cues) that come from person-to-person interactions be maintained with digital technology interventions?

to question the operational distinctiveness of 'goals' and 'task'. The psychometric evaluation of the Working Alliance Inventory (based on Bordin's ${ }^{9}{ }^{10} \mathrm{WA}$ ) suggested that these concepts were highly interrelated, ${ }^{29}$ while a more recent psychometric evaluation found that goals and task did not emerge as distinct factors. ${ }^{43}$

\section{Meaning of the study: possible explanations and implications for clinicians and policymakers}

Our findings address, at least in part, 3 of the 10 clinical and research priorities of digital technology in mental healthcare identified by people with lived experience of mental health conditions, carers and health and social care practitioners (see box 1) ${ }^{8} \mathrm{WA}$, a common element of psychotherapy appears to be both relevant and important in a b-CBT intervention for depression. Human delivery was reported as central to the maintenance of empathy, gestures and non-verbal cues, in which the PWP's role in b-CBT may be especially helpful in establishing the bond, and developing and maintaining the client's engagement through responsive support (Q8). Participants noted that both modes of delivery collaboratively contributed to the building of the alliance through distinctive pathways. While human support is perceived as 'responsive' and 'meaningful', digital delivery appears to promote autonomy and self-directed discovery (eg, accessibility and self-directed) which may be important for maintaining WA across 'goal' and 'task' activities (eg, ease of use, interactivity of digital program and aesthetic appeal). Our findings suggest that the removal of human support, may increase the risk of therapeutic ruptures and disengagement (Q1 and Q3). The findings of our study can be used to promote WA in technological design and clinical practice, thereby promoting engagement to b-CBT interventions for depression, and guiding the effective deployment of PWP and digital program resources.

\section{Unanswered questions and future research}

We propose four directions for future research. First, while our findings outline WA demands in b-CBT, it is unknown if fulfilling such demands will lead to positive clinical change. Future research should aim to investigate if self-reported WA as defined by our conceptual framework, predicts psychotherapy outcome. Second, WA 
should be further explored across different computerised programs, clinical groups, higher-intensity interventions and other digital technologies (eg, virtual experiences, gamification and text-based interventions) intended for use within a blended format, especially in relation to understanding how the demands of different digital technologies shape 'usability heuristics'. Third, our findings can be used to inform the design of behavioural intervention technology theories, as a means of enhancing engagement and adherence to digital components of blended interventions for mental health conditions. Fourth, given that digital technologies hold significant potential for bridging the gap in mental healthcare in low-resource settings, ${ }^{44}$ future research should examine WA in digital mental health interventions in non-Western cultures and settings.

\section{Author affiliations}

${ }^{1}$ Faculty of Epidemiology and Population Health, London School of Hygiene and Tropical Medicine, London, UK

${ }^{2}$ School of Psychology, University of Sussex, Brighton, UK

${ }^{3}$ Health Service and Population Research Department, King's College London, London, UK

${ }^{4}$ Department of Psychiatry, Oxford University, Oxford, UK

${ }^{5}$ Health Services Research and Policy, London School of Hygiene and Tropical Medicine, London, UK

\section{Twitter Asmae Doukani @asmae.doukani}

Acknowledgements The authors would like to thank the E-compared trial for supporting the study and the 11 patient advisors whose input shaped the methodology of the project, including Abé Chekh-Dove El-Ghassani, Michael Clarke, Paul H Ware, Dr Sarah Markham and Tibby Stodel. The authors would like to thank Dr Thomas Kabir from the McPin Foundation for his guidance on involving patient advisors, Dr Nicki Thorogood who provided guidance on the participant qualitative interviews methodology and to Shumaila Usmani who helped facilitate and transcribe the patient involvement focus group interviews.

Contributors $A D$ developed the concept of the work. Patient involvement shaped the focus of the research. AD led all aspects of patient involvement. AC-P and SS assisted with the patient involvement focus groups. The design and analysis of the patient involvement focus groups was contributed to by SS, JM-M, CF and RA. $A D, C F$ and SS significantly contributed to the design of the qualitative participant interview. $\mathrm{AD}$ led all aspects of data collection, analysis and interpretation. CF and DM analysed a portion of the data independently. The iterative development of the conceptual framework was led by $\mathrm{AD}$, overseen by DM and RK and contributed to by CF, RA, and AC-P. AD prepared all iterations of the manuscript, with significant contributions from RK, CF, DM, RA, JM-M, SS and AC-P.

Funding This work was supported by the E-compared trial, which was funded by the European Commission's Seventh Framework Programme (Health), grant agreement number 603098. JM-M is supported by the Wellcome Trust Grant (104908/Z/14/Z).

\section{Competing interests None declared}

Patient consent for publication Not required.

Ethics approval The project was approved by the Health Research Authority's Ethics Committee on 17 April 2015 (REC reference: 15/LO/0511) and the London School of Hygiene and Tropical Medicine Research Ethics Committee on 9 June 2015 (Ethics Ref: 9409).

Provenance and peer review Not commissioned; externally peer reviewed.

Data availability statement Data are available on reasonable request.

Open access This is an open access article distributed in accordance with the Creative Commons Attribution Non Commercial (CC BY-NC 4.0) license, which permits others to distribute, remix, adapt, build upon this work noncommercially, and license their derivative works on different terms, provided the original work is properly cited, appropriate credit is given, any changes made indicated, and the use is non-commercial. See: http://creativecommons.org/ licenses/by-nc/4.0/.

\section{ORCID iDs}

Asmae Doukani http://orcid.org/0000-0001-8629-2953

Jesús Montero-Marin http://orcid.org/0000-0001-5677-1662

Sarah Smith http://orcid.org/0000-0002-2013-6963

\section{REFERENCES}

1 OECD/EU. Health at a glance: Europe 2018: state of health in the EU cycle. Paris: OECD Publishing, 2018. https://doi.org/10.1787/health glance_eur-2018-en\%0Ahttps://www.oecd-ilibrary.org/social-issuesmigration-health/health-at-a-glance-europe-2018_health_glance_ eur-2018-en

2 WHO. Mental health: new understanding, new hope [online]. Geneva: World Health Organization, 2001. https://www.who.int/whr/2001/en/

3 WHO. Services and deinstitutionalization [online]. World Health Organization, 2019. http://www.euro.who.int/en/health-topics/ noncommunicable-diseases/mental-health/priority-areas/servicesand-deinstitutionalization

4 Fairburn CG, Patel V. The impact of digital technology on psychological treatments and their dissemination. Behav Res Ther 2017;88:25

5 Kleiboer A, Smit J, Bosmans J, et al. European comparative effectiveness research on blended depression treatment versus treatment-as-usual (E-COMPARED): study protocol for a randomized controlled, non-inferiority trial in eight European countries. Trials 2016;17:387.

6 van Ballegooijen W, Cuijpers P, van Straten A, et al. Adherence to Internet-based and face-to-face cognitive behavioural therapy for depression: a meta-analysis. PLoS One 2014;9:e100674.

7 Cavanagh K, Herbeck Belnap B, Rothenberger SD, et al. My care manager, my computer therapy and me: the relationship triangle in computerized cognitive behavioural therapy. Internet Interv 2018;11:11-19.

8 Hollis C, Sampson S, Simons L, et al. Identifying research priorities for digital technology in mental health care: results of the James Lind alliance priority setting partnership. Lancet Psychiatry 2018;5:845-54.

9 Bordin ES. The generalizability of the psychoanalytic concept of the working alliance. Psychother Res Practice 1979;16:252-60.

10 Bordin ES. Theory and research on the theraputic working alliance: New directions. In: Horvath AO, Greenberg Leslie S, eds. The working alliance: theory, research, and practice. New York: John Wiley \& Sons, 1994: 13-37.

11 Lambert MJ. Psychotherapy outcome research: implications for integrative and eclectic therapists. In: Norcross JC, Goldfried MR, eds. Handbook of psychotherapy intergration. 1st edn. New York: Basic Books, 1992.

12 Norcross JC, Lambert MJ. Psychotherapy relationships that work II. Psychotherapy 2010;48:4-8.

13 Cameron SK, Rodgers J, Dagnan D. The relationship between the therapeutic alliance and clinical outcomes in cognitive behaviour therapy for adults with depression: a meta-analytic review. Clin Psychol Psychother 2018;25:446-56.

14 Sucala M, Schnur JB, Constantino MJ, et al. The therapeutic relationship in e-therapy for mental health: a systematic review. $J$ Med Internet Res 2012;14:e110.

15 Vernmark K, Hesser H, Topooco N, et al. Working alliance as a predictor of change in depression during blended cognitive behaviour therapy. Cogn Behav Ther 2019;48:299

16 Kooistra L, Ruwaard J, Wiersma J, et al. Working alliance in blended versus face-to-face cognitive behavioral treatment for patients with depression in specialized mental health care. J Clin Med 2020;9:347.

17 Pihlaja S, Stenberg J-H, Joutsenniemi K, et al. Therapeutic alliance in guided internet therapy programs for depression and anxiety disorders - a systematic review. Internet Interv 2018;11:1-10.

18 Berger T. The therapeutic alliance in Internet interventions: a narrative review and suggestions for future research. Psychother Res 2017;27:511-24.

19 Barazzone N, Cavanagh K, Richards DA. Computerized cognitive behavioural therapy and the therapeutic alliance: a qualitative enquiry. Br J Clin Psychol 2012;51:396-417.

20 Berry K, Salter A, Morris R, et al. Assessing therapeutic alliance in the context of mHealth interventions for mental health problems: development of the mobile Agnew relationship measure (mARM) questionnaire. J Med Internet Res 2018;20:e90. 
21 Torous J, Jän Myrick K, Rauseo-Ricupero N, et al. Digital mental health and COVID-19: using technology today to accelerate the curve on access and quality tomorrow. JMIR Ment Health 2020; 7:e18848.

22 Warraich HJ, Califf RM, Krumholz HM. The digital transformation of medicine can revitalize the patient-clinician relationship. NPJ Digit Med 2018;1:49.

23 Hayes H, Buckland S, Tarpey M. Briefing notes for researchers: public involvement in NHS: public health and social care research, 2012. http://scholar.google.com/scholar?hl=en\&btnG=Search\&q= intitle:Briefing+notes+for+researchers:+public+involvement+in+ $\mathrm{NHS}$,+public+health+and+social+care+research\#2

24 Krueger RA. Focus groups : a practical guide for applied research. 2nd edn. London: Sage, 1994.

25 Staniszewska S, Brett J, Simera I, et al. GRIPP2 reporting checklists: tools to improve reporting of patient and public involvement in research. BMJ 2017;358:j3453.

26 Kroenke K, Spitzer RL, Williams JB. The PHQ-9: validity of a brief depression severity measure. J Gen Intern Med 2001;16:606-13.

27 Lecrubier Y, Sheehan DV, Weiller E, et al. The mini international neuropsychiatric interview (MINI). A short diagnostic structured interview: reliability and validity according to the CIDI. Eur psychiatr 1997;12:224-31.

28 Corbin J, Strauss A. Basics of qualitative research: techniques and procedures for developing grounded theory. 4th edn. London: SAGE Publications, 2008.

29 Horvath AO, Greenberg LS. Development and validation of the working alliance inventory. J Couns Psychol 1989;36:223-33.

30 Beck Institute. What is cognitive behavior therapy [online], 2016. Available: https://beckinstitute.org/get-informed/what-is-cognitivetherapy/ [Accessed $16 \mathrm{Jul}$ 2019].

31 Green J, Thorogood N. Qualitative methods for health research. 3rd edn. London, 2014.

32 Braun V, Clarke V. Using thematic analysis in psychology. Qual Res Psychol 2006;3:101.

33 Llewelyn S, Macdonald J, Aafjes-van Doorn K. Process-outcome studies. In: APA handbook of clinical psychology: theory and research. Washington: American Psychological Association, 2016: Vol 2. 451-63. http://content.apa.org/books/14773-020

34 O'Brien BC, Harris IB, Beckman TJ, et al. Standards for reporting qualitative research. Academic Medicine 2014;89:1251.

35 Cahill J, Barkham M, Hardy G, et al. A review and critical appraisal of measures of therapist-patient interactions in mental health settings. Health Technol Assess 2008;12

36 IAPT. Analysis document for improving access to psychological therapies (IAPT) referral rates by sex and age and sex and ethnicity [online]. London, 2017. Available: https://www.england.nhs.uk/ publication/improving-access-to-psychological-therapies-servicesanalysis/

37 Montero-Marín J, Prado-Abril J, Botella C, et al. Expectations among patients and health professionals regarding web-based interventions for depression in primary care: a qualitative study. J Med Internet Res 2015;17:e67.

38 Rogers CR. The necessary and sufficient conditions of therapeutic personality change. J Consult Psychol 1957;21:103

39 Kawamichi H, Yoshihara K, Sasaki AT, et al. Perceiving active listening activates the reward system and improves the impression of relevant experiences. Soc Neurosci 2015;10:26

40 Schuller D, Schuller BW. The age of artificial emotional intelligence. Computer 2018;51:46

41 Knowles SE, Toms G, Sanders C, et al. Qualitative meta-synthesis of user experience of computerised therapy for depression and anxiety. PLoS One 2014;9:e84323.

42 Mohr DC, Cuijpers P, Lehman K. Supportive accountability: a model for providing human support to enhance adherence to eHealth interventions. J Med Internet Res 2011;13:e30.

43 Hatcher RL, Barends AW. Patients' view of the alliance in psychotherapy: exploratory factor analysis of three alliance measures. J Consult Clin Psychol 1996;64:1336

44 Naslund JA, Aschbrenner KA, Araya R, et al. Digital technology for treating and preventing mental disorders in low-income and middle-income countries: a narrative review of the literature. Lancet Psychiatry 2017;4:486-500. 